Voix et Images

voixetimages

\title{
Entretien avec Nicole Brossard
}

Karim Larose et Rosalie Lessard

Volume 37, numéro 3 (111), printemps-été 2012

Nicole Brossard

URI : https://id.erudit.org/iderudit/1011950ar

DOI : https://doi.org/10.7202/1011950ar

Aller au sommaire du numéro

\section{Éditeur(s)}

Université du Québec à Montréal

\section{ISSN}

0318-9201 (imprimé)

1705-933X (numérique)

Découvrir la revue

Citer ce document

Larose, K. \& Lessard, R. (2012). Entretien avec Nicole Brossard. Voix et Images, 37(3), 13-29. https://doi.org/10.7202/1011950ar d'utilisation que vous pouvez consulter en ligne.

https://apropos.erudit.org/fr/usagers/politique-dutilisation/ 


\title{
ENTRETIEN AVEC NICOLE BROSSARD
}

\author{
$+++$ \\ KARIM LAROSE \\ Université de Montréal \\ ROSALIE LESSARD \\ Université du Québec à Montréal
}

VoIX ET IMAGES Dans votre cercle familial, comment les livres et la culture étaient-ils considérés? Quelle part leur réservait-on?

NICOLE BROSSARD Ils étaient bien perçus mais je ne me souviens pas qu'ils aient eu une présence importante dans le quotidien.

VoIX ET IMAGES De façon plus spécifique, quelle place la poésie tenait-elle dans votre enfance? Étiez-vous déjà en contact avec l'une ou l'autre de ses formes écrites?

NICOLE BROSSARD Elle était absente de la vie quotidienne. C'est en fait par le biais de séries diffusées à la radio que j'ai entendu les premiers noms de poètes québécois, celui de Jovette Bernier par exemple. Tout compte fait, il m'aura fallu attendre l'adolescence pour établir un premier contact avec la poésie de Chateaubriand, de Lamartine, de Villon, dont j'appréciais le petit côté délinquant, et par la suite sont venus Rimbaud et Verlaine. À l'école, j'ai découvert le plaisir que produisait la lecture. $\mathrm{J}$ 'aimais les questions, la réflexion qui, en classe, entouraient la lecture commune de textes. Mes premières années n'ont pas vraiment été marquées par l'univers de la poésie, mais par celui de la politique qui, lui, était au cœur des discussions familiales. Les déclarations de Maurice Duplessis soulevaient chez nous la colère et la révolte, et donnaient lieu à de grands débats. On le détestait avec ardeur.

voIX ET IMAGES $Y$ avait-il chez vous, avant même que vous ne commenciez à écrire, un imaginaire associé au monde de l'écriture?

NICOLE BROSSARD Il y avait peu d'images, et certainement aucune mythologie associée à l'écriture. Ma vie intérieure était davantage tournée vers l'acte de penser. C'était surtout lors des parcours entre l'école et la maison, dans ces moments de liberté et de silence, où je marchais le nez en l'air, en flânant, que j'ai pris l'habitude de la réflexion et du questionnement existentiel, dans un bonheur toujours lié aux sensations du corps en mouvement, au plaisir vital, à l'emportement de l'esprit, qui trouvait dans la sensualité son élan. Ce sont les moments forts de mon enfance. Cette rêverie "spirituelle» me semble avoir été initiatrice, chez moi, d'un certain rapport à l'urbanité et, plus largement, au réel, dans lequel j'étais, même toute jeune, fortement ancrée.

voIX ET IMAGES Vous souvenez-vous d'un moment où, à l'adolescence, vous avez pensé : «l'écriture est pour moi»? 
NICOLE BROSSARD À cet âge, rien n'était aussi clair, et cette question, si je me la suis posée, ce n'est sans doute pas en ces termes-là. J'écrivais des textes de quelques lignes que je n'aurais certainement pas qualifiés d' «écriture", ni de journal intime, à peine de poèmes. Ils provenaient surtout d'un franc plaisir à combiner les mots, et traduisaient les impressions assez anodines produites sur moi par la nature. C'était bien sûr le début de la formation d'un sujet: avec les mots, mais sans les livres. Et des clichés aussi qui, en somme, font partie de l'apprentissage premier.

voIX ET IMAGES Sans les livres mais avec les mots: pourrait-on dire que c'est une certaine conscience linguistique qui vous a menée à la poésie?

NICOLE BROSSARD La langue, on la parlait, et on savait qu'on allait se faire corriger si on ne la parlait pas bien. Mon rapport à la langue se limitait à une conscience normative. Avant les années 1960 et l'éveil politique du Québec, je n'avais pas de relation particulièrement affective à la langue. Vers 1961-1963, puis avec Parti pris, donc avec la question du joual et celle de l'aliénation, j'ai développé une réflexion plus critique sur la langue dans la foulée d'Albert Memmi et de Frantz Fanon. Et plus tard, avec le féminisme, la réflexion s'est poursuivie passionnément.

voIX ET IMAGES Quelles sont les premières œuvres littéraires qui vous ont interpellée?

NICOLE BROSSARD D'abord Rilke avec les Lettres à un jeune poète et Les élégies de Duino, et Camus avec Noces et L'été. Puis le choc vint, en 1965, avec la parution de Prochain épisode. Le roman d'Aquin a été pour moi le signe fort et stimulant d'un renouveau vital dans notre littérature. L'intensité et la tension créées par Aquin me parlaient en direct, si je puis dire. Je suivais à l'Université de Montréal le cours du soir de Réginald Hamel, qui nous a véritablement sensibilisés à la construction de l'histoire littéraire québécoise, qui avait eu tendance à exclure les œuvres transgressives ou originales, et à la question cruciale des choix idéologiques qui ont façonné notre mémoire culturelle. Avec lui, on a détesté corps et âme les Mandements de $\mathrm{M}^{\mathrm{gr}}$ Bourget! Et on a redécouvert Charles Gill, Jean-Aubert Loranger, La scouine, des voix qui étaient passées à la trappe et qu'on voulait faire entendre. On n'a cependant pas retrouvé, à l'époque, les voix de Medjé Vézina, d'Éva Senécal ou de Simone Routier. Cela, il a fallu le faire nous-mêmes plus tard.

voIX ET IMAGES Réginald Hamel était-il proche des membres du comité de rédaction de la revue La Barre du jour, que vous avez contribué à fonder en 1965 ?

NICOLE BROSSARD Oui. Mais entendons-nous : on ne parlait pas avec lui des propositions théoriques qu'on retrouvait dans les revues françaises Change et Tel Quel. Réginald Hamel discutait surtout avec nous d'histoire littéraire et de textes québécois, deux sujets qui étaient au cœur de notre réflexion sur la littérature. L'un des objectifs fondamentaux à La Barre du jour était justement de faire entendre des voix oubliées, écartées de l'histoire littéraire par conformisme ou conservatisme.

voIX ET IMAGES Quelles découvertes littéraires ont marqué votre venue à l'écriture, qui mènera à la publication d'Aube à la saison en 1965 ?

NICOLE BROSSARD Les lectures sont venues d'une rencontre importante, qui est celle, en 1963, de ce poète et grand lecteur de poésie qu'était Michel Beaulieu. En discutant avec lui, j'en suis venue à réfléchir plus spécifiquement à la poésie et à multiplier les lectures. Ces lectures, je les ai faites dans le cadre de l'Université de Montréal, en 
effervescence à ce moment, qu'on pense aux Presses de l'A.G.E.U.M. ou au journal étudiant Le Quartier latin, auquel j'ai collaboré en tant que critique d'art et de poésie, à la demande de Michel Beaulieu, et aussi en publiant dans ce journal des poèmes, après y avoir été invitée, une fois encore, par Beaulieu. En 1965, il me sollicite à nouveau, pour une publication officielle cette fois, et qui donnera le collectif Trois. Puis après avoir fondé la maison d'édition de l'Estérel, Beaulieu me propose d'y publier des poèmes, ce qui produira Mordre en sa chair (1966) et L'écho bouge beau (1968). Et, peu avant 1970, c'est Gaston Miron qui me demande de soumettre des textes à l'Hexagone. J'y publierai alors Suite logique (1970).

voix et Images Pour vous, l'écriture poétique s'est donc présentée comme une réponse à des appels de textes? Ce n'est pas banal! Écrire des recueils à partir d'invitations...

NICOLE BROSSARD Entre 1963 et 1965, il y avait un tel bouillonnement à l'université, et la littérature, la poésie surtout, entrait dans ma vie par la lecture, les discussions, le goût des projets, le plaisir de la découverte de nouveaux auteurs, érotiques, scandaleux, fous, etc. Mon désir de poésie a pris naissance dans ce contexte, mais il était aussi fortement lié au plaisir de l'acte d'écriture lui-même et de l'effet, pas seulement émotif, mais tout autant intellectuel, des mots. Quand j'écrivais, j'aimais l'être qui écrivait.

VoIX ET IMAGES Est-ce que, comme vous l'avez raconté à propos de votre enfance, on pourrait dire que chez vous l'écriture n'est pas venue des livres, mais bien de la vie elle-même, de l'émulation ou du contact direct avec des poètes et des éditeurs?

NICOLE BRosSARD Pour une part, oui, l'écriture s'est pour moi formée à même la vie sociale. Les lancements, les cours, les réunions, les soirées, où j'ai fréquenté les gens de La Barre du jour, de Parti pris, Paul-Marie Lapointe ou Gaston Miron, ont eu leur importance et ont occasionné de nombreuses discussions au cours desquelles nous ne nous contentions pas de refaire la littérature : nous refaisions le monde, avec beaucoup d'intensité et d'énergie (nous ne savions pas que c'était le fait de chaque génération!). Tout cela a été très rapide.

VoIX ET IMAGES Il ne faudrait donc pas prendre littéralement ces vers d'Aube à la saison : «La parole me fut donnée ${ }^{1}$ » ? La poésie ne viendrait pas pour vous comme une épiphanie, ou comme un arrachement?

NICOLE BRosSARD Non, en effet. Ce que vous appelez ma "venue à l'écriture» s'est fait tout naturellement. Dans Aube à la saison et Mordre en sa chair, on entend d'ailleurs l'influence des écrivains d'alors. Le flot d'images et de métaphores, cultivées dans la poésie de l'époque, s'inscrit clairement dans ces deux premiers livres.

voIX ET IMAGES On entend aussi quelques échos hébertiens dans vos premiers recueils. Était-ce une lecture importante?

NICOLE BROSSARD Oui. Ā part Gemma Tremblay, Suzanne Paradis et les poètes haïtiens et surréalistes, au lyrisme généreux, il y avait Le tombeau des rois et Mystère de la parole, une œuvre saisissante, énigmatique, qui témoignait des dangers qui se logent

$$
++
$$

1 Nicole Brossard, "Aube à la saison», Le centre blanc. Poèmes 1965-1975, Montréal, l'Hexagone, coll. «Rétrospectives», 1978, p. 21. 
dans les poèmes, et de l'étrange voyage qu'on peut y vivre quand la charge symbolique qui les habite reconfigure l'usage du poétique.

voIX ET IMAGES Dans quel contexte avez-vous fait la rencontre des écrivains avec lesquels, en 1965, vous avez fondé La Barre du jour ${ }^{2}$ ? Et comment, vous et le cercle d'amis de l'Université de Montréal avec lesquels vous alliez fonder la revue, avezvous fait le saut de l'université à la scène littéraire?

NICOLE BROSSARD Il y a d'abord eu cette rencontre de quatre univers: Roger Soublière, très près de la contre-culture, Marcel Saint-Pierre, qui lisait surtout les surréalistes français et québécois et apportait avec lui de nouveaux textes - les poèmes de René Daumal, de Joë Bousquet et Refus global, par exemple -, Jean Stafford, qui tenait un discours sociologique, et moi, qui étais surtout intéressée par la création. La fondation de la revue est née de notre désir de donner un lieu à la modernité québécoise et de notre fort sentiment qu'il était indispensable de relire des textes québécois négligés. Parallèlement à notre activité au sein du comité de la revue, il nous a semblé tout naturel de publier des livres. C'est ainsi que paraîtront L'anti-can (1969) de Roger Soublière et mon recueil Le centre blanc (1970), tous les deux avec des dessins de Marcel Saint-Pierre.

voIX ET IMAGES Et le saut, de la vie d'étudiant à celle de jeune écrivain?

NICOLE BROSSARD En fait, il n'y a pas eu de saut. Cela s'est fait tout naturellement. Pas vraiment de coupure entre le milieu universitaire et culturel. On écrivait, on discutait et on publiait. On inventait notre désir jusqu'à ce qu'il se transforme en livre. voIX ET IMAGES Mais concrètement, de quoi vivez-vous quand s'achèvent les études à l'Université de Montréal?

NICOLE BROSSARD J'ai poursuivi des études à l'UQAM. Pour obtenir un bout de papier, j'y ai fait un diplôme en pédagogie, qui m'a prodigieusement ennuyée - ça ne devait vraiment pas être mon avenir! J'ai malgré tout enseigné deux ans dans une école secondaire, puis j'ai obtenu des bourses, me permettant, par la suite, de consacrer mon temps à l'écriture. Ce sont les deux seules années de ma vie où j'ai travaillé dans un cadre.

voIX ET IMAGES Y a-t-il eu, à La Barre du jour, une tentation du surréalisme (vous préparerez notamment des numéros spéciaux sur Gauvreau et sur Giguère) ou, au contraire, les positions et l'esthétique surréalistes vous étaient-elles lointaines? Vous êtes-vous rapidement dirigés du côté de Tel Quel?

NICOLE BROSSARD Pour notre groupe, cela a vite été une écriture, disons du non-sujet, une écriture autoréférentielle qui a pris le devant. Le surréalisme me semblait un peu suspect, je dois dire: le manifeste, d'abord, puis l'arrogance de Breton, la vanité d'Aragon, que j'avais entendu au Sel de la semaine, l'émission de Fernand Seguin, la hiérarchisation du mouvement et l'autoritarisme de son grand pape. Malgré cela, je me sentais ambivalente à l'égard des surréalistes français. J'ai toujours respecté l'intelligence du texte et du rêve qu'on trouve chez eux, leur conception très libertaire de l'écriture et la grande beauté des images qui découlaient de cette pratique très

$$
+++
$$

2 Sur les détails de la fondation de La Barre du jour, voir «Entrevue avec Nicole Brossard, Roger Soublière et Marcel Saint-Pierre», Voix et Images, vol. X, n² 2, hiver 1985, p. 77-81. 
libre; néanmoins, ça jouait dur au sein du mouvement à l'égard des femmes et, même avant que j'articule une réflexion féministe, les femmes-objets présentes dans les œuvres surréalistes me posaient problème. Les écrivains surréalistes étaient des interlocuteurs; le mouvement, lui, provoquait en moi un réflexe de résistance. C'est une autre modernité, une autre posture dans le texte, moins narrative, qui m'a davantage rejointe. Soit dit en passant, le machisme telquellien n'avait rien à envier à celui des surréalistes.

VoIX ET IMAGES Claude Gauvreau, lui, était-il présent pour vous?

NICOLE BROSSARD Ses textes étant introuvables, c'est surtout l'homme que nous avons appris à connaître. On le croisait au restaurant La Hutte, avec Miron. On échangeait avec eux. Ils racontaient. Refus global, pour moi, a été un texte marquant. Gauvreau ouvrait, lui, sur tout un monde...

VoIX ET IMAGES Quelle était votre position par rapport à ce que faisaient les écrivains de Parti pris?

NICOLE BROSSARD On départageait le Parti pris politique, ironique, contestataire, dont l'existence nous apparaissait importante, et le Parti pris littéraire. Tout cela se passait un peu en parallèle. J'ai beaucoup aimé La ville inhumaine de Laurent Girouard, la colère aussi qui se dégageait du Cassé de Jacques Renaud, le troublant L'inavouable de Paul Chamberland, mais avec le travail de Jasmin, et la fixation dans le joual, notre admiration s'est émoussée et s'est, en tout cas, transformée en indifférence, voire en désaccord esthétique. Le joual, le joual colérique, si je puis dire, s'enlisait dans une langue de l'impuissance, à partir de laquelle on ne pouvait pas vraiment penser le monde.

VoIX ET IMAGES Les débuts de la période formaliste, en 1970, voient la publication de deux recueils en un an, hantés par un imaginaire de la fin, par la conscience de la limite de l'expression poétique et la proximité de la prose. Car 1970 est aussi l'année où paraît Un livre, votre premier roman... Comment le formalisme a-t-il modifié votre pratique de l'écriture?

NICOLE BRosSARD Ma poésie naît des mots eux-mêmes (leur résonance, leur charge symbolique, leur sonorité, leur agencement). Ce que j'appelais le «neutre» et qu'on aurait pu associer au formalisme était en fait cette liberté d'une écriture qui ne s'appuie sur rien d'autre que les mots comme point de départ et dont le départ n'est pas déjà peuplé par un récit, un sentiment précis. On dirait parfois que j'apprends autant des mots que de la vie et que je jouis des deux quand ils font la paire. On n'écrit vrai qu'en position de vulnérabilité, mais on n'écrit rare que dans le plaisir des mots. Avec le formalisme, je pouvais expérimenter, je pouvais ouvrir des portes, tout était possible, la langue avait de l'imagination pour moi. Elle prenait les devants. Je pouvais m'appuyer sur sa puissance génératrice. Le formalisme, pour cette raison, a été important comme expérience d'une retenue dans l'acte d'écriture. Je n'ai jamais été sous l'emprise d'une théorie formaliste, parce que pour moi la théorie vient après l'expérience et permet de comprendre, a posteriori, le processus de création. La théorie ne m'a pas censurée, limitée, au contraire. Je n'ai pas honte, aujourd'hui, du mot «formalisme», qui m’a permis d'aller du côté du fragment, du présent fragmenté, qu'on retrouve déjà dans L'écho bouge beau et dans Suite logique. Avec Le centre blanc, ce fut autre chose: la prise de conscience d'un indicible, et la peur de ne plus pouvoir 
écrire. C'était un travail d'écriture avec contraintes mathématiques, travaillé par les mots «corps », «souffle», "mort», et qui m’a appris que la littérature est ce qui permet à l'infini un jeu de permutation du sens entre ces deux extrêmes de l'expérience humaine que sont la jouissance et la douleur absolues.

voIX ET IMAGES Dès les premiers recueils, vous avez multiplié les lieux de publication. Au fil des ans, les maisons d'édition se succèdent: l'Estérel, l'Hexagone, les Éditions d'Orphée, de l'Aurore, les Écrits des Forges, le Noroît... Qu'est-ce qui explique une telle diversité de lieux pour la poésie, contrairement au roman, par exemple?

NICOLE BRosSARD Vous voulez dire que je ne suis pas une femme fidèle, c'est ça! voIX ET IMAGES Certainement pas! Mais est-ce que le changement de maison est dû aux politiques éditoriales, à un désir de migrer, de passer d'une identité éditoriale à une autre, ou est-ce plutôt le hasard des rencontres qui vous a guidée?

NICole BRosSARD C'est en partie un désir de déplacement, de nouveau départ, mais $c^{\prime}$ est aussi ma passion pour les beaux livres qui m'a fait changer d'éditeur au fil du temps. Cette infidélité s'explique aussi par le fait que je n'ai jamais développé, avec un éditeur de poésie, de dialogue approfondi et continu autour de mes textes. Ce sont mes traductrices et mes traducteurs qui ont joué ce rôle de questionnement et de dialogue. Pour le roman, en revanche, je garde un beau souvenir du travail de lecture fait par Pierre Filion, au moment de la publication de La capture du sombre (2007).

voIX ET IMAGES Abordons maintenant la question du féminisme, si vous le voulez bien. Dès le milieu des années 1970, vous avez été l'une des premières écrivaines dans l'histoire littéraire québécoise à vivre de poésie et à porter, dans vos textes, une conscience féministe. Qu'est-ce que cela a représenté pour vous, à la fois concrètement et symboliquement?

NICOLE BROSSARD La conscience féministe permet de passer d'un monde où les femmes sont invisibles ou, quand elles sont visibles, uniquement charnelles et moralement coupables de tout, à un monde où les femmes deviennent sujets, centre d'intérêt, et sont valorisées comme individus et comme groupe. Symboliquement le passage est considérable car il fait exploser la version patriarcale de la réalité et des mœurs et, concrètement, il fait éclater la patience endurante des femmes, ce qui a pour effet de les rendre plus attentives à leurs désirs et à leurs besoins, de les rendre solidaires des autres femmes et de les motiver à la lutte pour leurs droits et différences. Comprendre pourquoi la moitié de l'humanité n'appartient pas à l'humanité, mais à un genre minorisé, est une des énigmes les plus effroyables et difficiles à résoudre, surtout quand cela s'accompagne d'intimidation, de mépris, voire de violence. Je voulais comprendre. Il y a donc eu la lecture des livres de Kate Millet, Ti-Grace Atkinson, Shulamith Firestone. Pour les collaboratrices des Têtes de pioche (revue lancée en 1976), les affinités idéologiques étaient du côté du féminisme politique américain alors que pour le cercle des écrivaines féministes, les affinités allaient vers des textes plus près de la psychanalyse. Pour ma part, c'est du côté américain que j'ai eu envie de regarder en codirigeant avec Luce Guilbeault le film Some American Feminists (1977). La nouvelle sociabilité et la solidarité vécues dans le mouvement féministe se sont sans doute traduites, dans mon écriture, par un recul du fragment. Intuitivement, je dirais que mon écriture s'est à ce moment modifiée, arrondie, adoucie, tout en se chargeant de l'énergie de la révolte et d'un désir radical de changement existentiel auquel l'amour lesbien 
n'était pas étranger. Le sens apparent (1980) et Amantes (1980) témoignent tout particulièrement d'une nouvelle sensibilité formelle plus près de la prose. Des mots tout simples ont ressurgi, ont pris une nouvelle configuration, une autre dimension symbolique: "peau», «sommeil», "spirale», «continent». La fiction, pour moi, avait le pouvoir d'engendrer du réel, du réel inédit, qui n'avait pas d'existence à l'intérieur de l'univers patriarcal. Elle amenait aussi à tirer de nouvelles conclusions, théoriques, sur le monde dans lequel nous vivions et à modifier le visage réel de notre société. Lancer sur la page quelques énoncés, prendre le risque d'affirmer quelque chose qui n'avait pas droit de cité, bousculait, à mon sens, la loi. Je crois que c'est de cette conviction qu'est venu mon désir d'écrire une fiction-théorie (L'amèr, 1976). Avec ce livre, j'ai appris à incorporer des bribes d'un réel éclipsé, des morceaux de la réalité concrète des femmes.

VoIX ET IMAGES A-t-il pu, aussi, être écrit dans un malaise par rapport aux genres institués, comme la poésie, incapables de rendre compte de votre réflexion féministe, parce qu'ils étaient du côté de la Loi et du sens commun, parce que les genres littéraires étaient des intermédiaires entre la Loi et les individus (les écrivains, les lecteurs)?

NICOLE BROSSARD Peut-être. L'éclatement du genre poétique, pour moi, s'est fait sans autre direction que celle qu'imposait la nécessité d'une version des faits au féminin et de déjouer linguistiquement les stratégies du mensonge patriarcal et de la logique sexiste. C'est ce questionnement qui a mené à l'éclatement des genres. C'est le tourment, la colère, le faux dualisme fiction/réalité, le trafic symbolique qui ont nécessité le glissement des genres les uns vers les autres et les uns contre les autres.

voIX ET IMAGES Formellement, L'amèr et Amantes sont des livres bien différents l'un de l'autre. Selon vous, sur quoi se fonde cette différence?

NICOLE BROSSARD La prose de L'amèr est reliée au quotidien et à la matière associée aux contraintes du réel liées à mon expérience de la maternité. Les deux ont trouvé leur forme naturelle dans la prose, plus réflexive, plus philosophique, mais aussi plus anecdotique. Justement, une petite anecdote sur la matière et le monde de la petite enfance. Il y a quelques années, une étudiante universitaire m'a fait part de ses recherches sur le manuscrit de L'amèr, et elle m'a confié avoir trouvé des miettes, des taches de nourriture sur le papier. La matière, avec un enfant, entre partout dans nos vies et même, littéralement, dans des manuscrits...

Amantes est un recueil de poèmes qui traduit l'énergie, la beauté, l'intégrité des amours lesbiennes ainsi que l'utopie qui en jaillit. Mais les choses ne sont pas si simples, et le partage, jamais aussi net. Le sens apparent, discursif et en prose, est pour moi traversé d'éclairs de poésie et même de lyrisme. Disons qu'à ce moment-là, en m'interrogeant sur l'énigme du genre féminin repoussé hors frontières du genre humain, je me suis trouvée à circuler entre les genres par besoin. Et cela, bien qu'exigeant, m'a aussi donné ce que Roland Barthes appelait «le plaisir du texte».

voIX ET IMAGES La poésie, comme espace marginal, vous semblait-il un lieu générique propre à exercer une lutte féministe?

NICOLE BRosSARD Non. Certainement pas propice à exercer une lutte féministe. Par contre, je dirais que le poème a permis de faire exister ce qui ne pouvait pas encore être formulé prosaïquement, c'est-à-dire idéologiquement. Il a aussi été le lieu privilégié de la rencontre du je et du nous, il a été et est toujours un creux intime de vie, tourment ou emportement. 
voIX ET IMAGES Dans vos entretiens, au fil des ans, vous avez insisté sur le plaisir que vous tirez de l'acte d'écrire. Y a-t-il un plaisir spécifique à l'écriture poétique, si on la compare à l'écriture romanesque?

NICOLE BROSSARD La poésie vibre toujours au présent. Quand on l'écrit. Quand on la relit. Elle a partie liée avec une intensité spécifique au plaisir des mots et à l'émotion, à la sensation qu'ils suscitent ainsi qu'au sens qu'ils suggèrent. Le roman se pense et se construit dans la durée, l'incertitude, l'endurance du coureur.

VoIX ET IMAGES Y a-t-il chez vous une douleur liée à l'écriture, une zone d'ombre, un soupçon? Dans une entrevue accordée à Michel Van Schendel et Jean Fisette, vous affirmiez notamment qu'écrire, «c'est toujours un prétexte pour continuer d'avoir l'air d'aller dans l'existence ${ }^{3} »$. S'agit-il là d'une remarque isolée, peu représentative? NICOLE BROSSARD La douleur serait, je crois, de consentir à ne plus écrire. Cela me fait penser à une citation de Saint-Denys Garneau que Benoît Jutras a mise en ligne sur Facebook: «Tout m'est prétexte à faire semblant de vivre, de penser, d'aimer. Si j'étais rigoureusement honnête, je ne lirais plus, je n'écrirais plus, ne verrais personne. Je

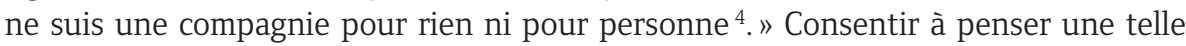
chose et à l'écrire me donne le vertige. Perdre la foi en l'écriture, pour un poète, c'est se couper du monde, non pas de la partie sombre du monde mais de son potentiel de joie. Si on doit désespérer, il vaut mieux le faire dans le poème lui-même car la langue recommence chaque fois - là. L'autrement dit relance l'énergie du monde.

Voix ET IMAGES À quel moment ressentez-vous la nécessité d'écrire de la poésie? NICOLE BROSSARD Il y a les moments forts de la vie, l'amour, la peine d'amour et le deuil, qui modifient le sens et déclenchent l'écriture poétique. Confronté à ces expériences, ce que fait naturellement un poète, c'est de trouver un nouveau sens. Et puis, il y a les lectures et les rencontres, avec des personnes ou des villes, avec leur beauté, qui peuvent moduler le goût du poème et de la poésie. C'est toujours du danger et de la passion, d'un état de tension, personnel ou collectif, que naît le désir de poésie. Et la poésie est fondatrice de ce qui est, au sens où elle fait entrer dans la langue la face cachée des désirs ou des désespoirs et préfigure souvent la posture sociale et historique d'un groupe d'appartenance. Avoir des poèmes en tête, les siens ou ceux des autres, c'est entretenir la mémoire collective.

VoIX ET IMAGES Y a-t-il eu, pour vous, des moments de découragement, d'impasse, à la Garneau? Comment avez-vous vécu les silences ou les demi-silences poétiques que vous avez traversés, notamment au milieu des années 1980 ?

NICole BRosSARD Je n'ai jamais eu le sentiment que je n'écrirais plus de poésie; par contre, j'ai éprouvé le sentiment que je n'écrirais plus, tout court, après Le centre blanc, Amantes et Le désert mauve (1987). À la suite de décharges énergétiques aussi intenses, la parole est plus lente à reprendre. L'effervescence met du temps à revenir. Par contre, je peux dire que je n'ai jamais imaginé ma vie sans poésie, encore moins aujourd'hui.

3 Michel Van Schendel et Jean Fisette, «Un livre à venir. Rencontre avec Nicole Brossard», Voix et Images du pays, vol. III, nº 1, septembre 1977, p. 4.4 Saint-Denys Garneau, Euvres, édition critique établie par Jacques Brault et Benoît Lacroix, Montréal, Presses de l'Université de Montréal, coll. «Bibliothèque des lettres québécoises», 1971, p. 542. 
C'est intéressant que vous ayez remarqué ces plages de silence; moi, je ne les ai pas perçues. Il y avait simplement la vie. Je refaisais le plein. Il n'y a pas eu arrêt de l'écriture, mais un déplacement vers les activités féministes, des voyages et l'amour, qui prenaient beaucoup de place. J'ai souvent dit que, devant un épuisement de l'écriture et la proximité du silence, il y a trois attitudes possibles: ou on fait le clown, ou on arrête d'écrire, ou on se suicide. Et il y a la quatrième voie, bien sûr, qui est de continuer d'écrire à un autre rythme, celui-là investi de silence. Il faut seulement espérer que la petite perle à mystère au fond de soi continue à scintiller et à nous intriguer.

VoIX ET IMAGES $Y$ a-t-il pour vous une énergie particulière liée aux voyages? Plusieurs de vos recueils plus ou moins récents sont associés à un art du voyage...

NICOLE BROSSARD Oui, en nous éloignant du confort, le voyage nous rend plus vigilants, nous oblige à raffiner nos antennes. C'est une ouverture constante aux autres, aux autres langues aussi, si on a l'élan pour les accueillir. C'est un espace existentiel très privilégié si on s'y abandonne car il offre la solitude, suscite des tensions, nous rend anonyme, analphabète et intrigué par notre présence au monde. Voyager sans flâner n'est pas voyager, tout comme écrire sans flânerie dans les pensées est impossible. J'aime que s'implantent en moi des paysages nouveaux, mes palmiers chéris de Key West ou de Dublin. Tous les écrivains savent qu'il faut voyager. Les poètes encore plus. VoIX ET IMAGES Dans les recueils centrés autour de l'expérience du voyage, avez-vous le sentiment d'un déplacement de la forme ou de la subjectivité, induit par le voyage lui-même?

NICOLE BROSSARD Je ne sais pas... Avec Je m'en vais à Trieste, il y avait la crainte de ne pas assez écrire, de faire carte postale, mais comme c'est un processus qui a couru sur dix ans, je crois qu'il témoigne aussi d'un travail de présence rendant compte du plaisir, de mes préoccupations et d'une obsession du temps et du lieu qui, somme toute, me plaît. Il est certain aussi que la subjectivité est toujours stimulée par des associations. Trieste: Joyce et Svevo, la Bora. Buenos Aires: Borges et tango. Jaipur: Palais des vents, rose, etc. En voyage, j'absorbe tout, je deviens une véritable éponge, surtout quand c'est dans le cadre d'une recherche en vue d'un projet. En fait ce n'est pas tellement la subjectivité qui se déplace mais son volume qui augmente la faculté de capter l'environnement autour de soi tout en étant attentif au moindre craquement de l'être en soi.

VOIX ET IMAGES Je m'en vais à Trieste, c'est non seulement un tour du monde, mais un panorama de l'histoire de l'art et des arts. Toutes les disciplines artistiques y sont convoquées.

NICOLE BROSSARD L'art est en effet un aimant qui attire vers les villes. D'une certaine manière, les villes existent en dehors du réel (le bruit, la circulation, la pauvreté) grâce à ceux et celles qui y ont rêvé et créé. Une ville c'est de la mémoire, du récit, une certaine luminosité déviée vers le présent. Ce qui me frappe le plus en voyage, c'est la façon dont la beauté, la lumière, l'architecture, les parcs, tout prend une dimension d'excessive attirance. J'aimerais refaire un deuxième Je m'en vais à Trieste. Quelle en serait la ville d'arrivée? Je ne sais pas encore mais j'en serai amoureuse.

VoIX ET IMAGES Un riche dialogue avec les arts visuels marque la partie la plus récente de votre production poétique, qu'on pense justement à Je m'en vais à Trieste en 2003, 
mais aussi à Installations en 1988 et au Musée de l'os et de l'eau en 1999. Comment et en quoi les arts visuels vous inspirent-ils?

NICOLE BROSSARD Il y a les œuvres mais aussi les lieux qui permettent de circuler entre elles, qui m'incitent à l'écriture. Le titre des œuvres, les notices qui les accompagnent, l'ambiance même du musée, le silence sont pour moi des stimuli très forts. C'est autant ce que je vois que ce que je cogite en regardant qui me fait écrire. Au fond, ce que dit l'art, c'est « vous ne savez pas regarder, voici pourquoi en regardant cette œuvre vous apprenez à percevoir autrement». Les arts visuels sont pleins de métaphores mais aussi ô combien remplis de narratifs qui reconfigurent la lumière tout autant que le politique, les objets tout autant que l'absence. Pour moi, les arts, c'est vivant comme le soleil et la nuit.

VoIX ET IMAGES En entrevue, vous avez confié que vos «manuscrits se corrigent au moment même où [vous] les [écrivez] ${ }^{5}$ ». Quelles sont aujourd'hui vos habitudes de réécriture? Écrivez-vous plusieurs versions d'un même poème?

NICOLE BROSSARD Maintenant, je corrige différemment, c'est-à-dire après avoir écrit et non pendant l'écriture. Mais la notion de version est complexe. Somme toute, je ne fais pas de «versions » d'un poème. Je corrige les poèmes, je les transforme comme un module. L'idée de version, en poésie, me semble poser la question de la sincérité. Fait-on plusieurs versions pour être plus sincère, plus précis ou pour formellement enrichir le poème? Quel rôle accorder, quelle place faire au mot surgi, par hasard, lapsus ou coquille et qui vit bien dans le texte tout en en changeant le cours? Que veut dire le mot «sincérité » en poésie? J'aime beaucoup ces questions-là qui mettent en parallèle les mots «version», «traduction», «transposition», «intrusion", « détournement».

VoIX ET IMAGES La réécriture n'étant jamais innocente, y a-t-il des choses que vous tentez d'éviter en corrigeant vos poèmes?

NICOLE BROSSARD La réécriture est liée à l'effet que produit sur moi, lectrice, le texte. Est-ce que le texte me surprend, me déroute, m'intrigue, m'émeut? Quel plaisir me donne-t-il ? La réécriture, en tout cas, n'est pas nécessairement liée à ce que je veux, à ce que je pense. J'essaie d'éviter l'ennui, et l'ennui découle souvent de la docilité grammaticale ou syntaxique.

VoIX ET IMAGES N'y a-t-il pas, dans votre écriture qui est marquée par la répétition - le leitmotiv dans Langues obscures (1992), les séquences, les reprises, les redites, la suite dans À tout regard (1989), la numérotation des poèmes - , une intégration de différents états d'un même énoncé? On ne pourrait pas voir, dans Piano blanc (2011) par exemple (Paupières 1, 2, 3), différentes variations d'une même question poétique?

NICOLE BROSSARD Il y a la question des angles et aussi celle de la variation. Nous répétons constamment nos gestes, nos paroles, nos idées. Je crois que la numérotation rencontre en moi un plaisir de la classification, de l'illusion de la précision et ce au moment même où les dérives du cœur prennent d'assaut le poème. J'aime aussi les chiffres, au même titre qu'une allée de palmiers ou de cyprès. Ils me donnent

$$
+++
$$

5 Michel Van Schendel et Jean Fisette, «Un livre à venir», p. 4. 
intrinsèquement du plaisir. Quant à Piano blanc, le recueil demeure encore pour moi une énigme. Il y a là des questions sur la prose et la poésie, et un bilan, avec notamment la référence à O.R., le personnage de mon premier roman, Un livre. Ce recueil pose des questions auxquelles je ne peux pas encore répondre. J'aime aussi qu'il en soit ainsi car cela suppose un enjeu important pour le futur.

VoIX ET IMAGES Concevez-vous la mise en recueil comme un travail de juxtaposition, de succession, de progression? À lire Musée de l'os et de l'eau, nous avions plutôt le sentiment de sections constituant des panneaux...

NICOLE BROSSARD Installations par exemple est construit par juxtaposition, page par page, alors que dans Musée de l'os et de l'eau, les textes sont juxtaposés de manière, comme vous dites, à donner l'impression de grands panneaux, ce qui donne un autre ton et un autre sentiment du temps. Dans Je m'en vais à Trieste, on pourrait sans doute parler de progression. Et avec Piano blanc on pourrait penser à une architecture dans le temps avec ouverture et clôture par deux verbes : «tressaillir» et « ruisseler ». Il arrive que l'on fasse un choix de rythme avant l'écriture d'un recueil; d'autres fois, le rythme s'impose à nous, un peu comme pour le choix des pronoms. Parfois j'opte pour des contraintes. Travailler avec des contraintes, c'est comme se baigner dans la mer au lieu de se baigner dans la piscine: la contrainte ouvre un espace, dans l'immensité de la langue, et nous force à trouver notre point d'ancrage, notre focus.

VoIX ET IMAGES La figure de l'ellipse est très présente dans votre œuvre. Y a-t-il, dans cette figure, une dimension éthique? Le choix de dire moins ou de ne pas tout dire vient-il d'une pudeur, d'une modestie du poète devant le langage? En entrevue, vous disiez: "la poésie, $c^{\prime}$ est l'ellipse par excellence ${ }^{6}$ ». Ce constat général nous ramène naturellement à votre propre travail poétique. Quel statut a pour vous l'ellipse?

NICOLE BROSSARD L'emploi de l'ellipse ne vient pas d'une éthique, d'une modestie ou d'une pudeur. Elle est plutôt liée à des préférences formelles et à une manière d'être dans l'existence. L'ellipse, c'est une façon de faire synthèse, un jeu d'absence et de présence qui permet de ne pas s'étendre dans «l'universel reportage» décrié par Mallarmé, d'aller à l'essentiel, à un saisissement du sens. En poésie, c'est l'effet de l'ellipse qui m'attire. Dans la vie, j'aime le raccourci. Tout cela a sans doute à voir avec ma relation à l'anecdote et au récit.

voIX eT IMAGES Dans Piano blanc, vous parlez des « infimes détails de récit/où on ne fait plus jamais la paix ${ }^{7}$ ». Y a-t-il là quelque chose d'un nouage possible entre poésie et récit? L'impossible paix...

NICOLE BROSSARD En poésie, les infimes détails peuvent susciter des images fortes, des métaphores. Dans la prose, ils sont souvent moteurs d'intrigue, de suspens, l'énigme qui retient notre attention, qui hante notre mémoire livresque. En ce sens, le génie des grands romans est dans le détail. Dans la vie, le détail peut passer inaperçu ou au contraire déclencher des émotions vives qui lient ou qui éloignent à tout jamais. Dans le récit, il est toujours signifiant. Oui, le détail peut constituer un nouage possible

$$
++
$$

6 Michel Van Schendel et Jean Fisette, «Un livre à venir», p. 5.7 Nicole Brossard, Piano blanc, Montréal, l'Hexagone, coll. «L'appel des mots», 2011, p. 75. 
entre poésie et récit. Il faut aimer les détails qu'on choisit, les cultiver, les regarder sous tous leurs angles, les étirer dans le temps comme des gestes infiniment précieux pour comprendre.

voıX ET IMAGES À l'instar de Miron, avez-vous déjà eu le sentiment d'écrire des nonpoèmes?

NICole BRosSARD Au début des années 1970, pendant l'application de la Loi des mesures de guerre, j'ai écrit un texte, "Tabarnak», reproduit dans Double impression (1984). Ce fut la même chose avec le poème "C'est étrange», écrit en 2003 et publié dans Après les mots (2007). Pour moi, le non-poème, c'est le poème qu'on ne voudrait pas écrire, mais qui surgit d'un débordement d'indignation, de colère et d'incompréhension devant une inimaginable violence. Le non-poème advient quand la poésie ne peut plus coexister avec la violence et l'injustice, quand le réel franchit un seuil inconcevable avec notre conception de «l'humain ». Avant de lire «C'est étrange» en public, je commence toujours par dire que je n'écris pas normalement de cette façon mais qu'un jour, n'en pouvant plus des rapaces de la société marchande et de la bêtise qu'elle cultive, il m'a fallu écrire ce texte. En fait, c'est parce que le non-poème est plein de sentiments forts qu'il nous réinsère dans l'aura du poème.

voIX ET IMAGES Dans les œuvres de la dernière décennie, vous adoptez un discours très critique à l'égard des sociétés contemporaines. Diriez-vous qu'il y a chez vous, dans Musée de l'os et de l'eau par exemple, la nécessité de mettre côte à côte la mémoire épique de la civilisation et la mémoire humiliée, clandestine des laissés-pourcompte de l'histoire? L'odyssée d'un côté, l'esclave non loin?

NICOLE BROSSARD Oui, un discours critique à l'égard d'une société marchande qui vend indistinctement femmes, enfants, gènes, forêts, pétrole, pommes vertes, etc., qui achète indistinctement les politiciens, les chercheurs, la nature, etc. Oui, un discours critique envers les cultures et les religions qui sortent les femmes de l'humanité à coups de mensonges et de viols. De l'autre côté, on aime rêver de L'odyssée, un œil sur le iPod, un autre sur les étoiles. J'aime la complexité de ce que nous sommes. C'est grâce à cette complexité que je garde espoir et curiosité.

VoIX ET IMAGES Un certain pessimisme ne vous épargne pas, en tout cas, le constat critique des problèmes fondamentaux de la société, comme on le voit dans ce poème d'Au présent des veines (1999): «je pourrais tout raconter/en français je veux dire/sans description seulement de mémoire/sans me tromper entre les fruits et les maladies/sans compter le nombre des morts/je pourrais très discrètement entre les paupières/rapprocher l'avenir en images fluides/ne rien faire pour détecter la barbarie $^{8}$ ».

NICOLE BRosSARD À l'origine de ce texte, je m'étais dit: écrivons un poème narratif. Et voilà, impossible d'échapper au quotidien qui, tout en nous offrant le confort de l'habitude, nous oblige aussi à "compter le nombre des morts». La barbarie est un mystère, n'est-ce pas, tout comme l'érosion des démocraties ou, disons, l'avènement des dictatures démocratisées.

$$
++
$$

8 Nicole Brossard, Au présent des veines, Trois-Rivières/Herborn/La Réunion, Écrits des Forges/Éditions Phi/Grand océan, 1999, p. 67. 
voix ET IMAGES Dans l'un de vos essais les plus connus, vous faisiez une distinction entre poésie engagée et poésie militante ${ }^{9}$. Qu ${ }^{\prime}$ est-ce qui distingue pour vous ce poème d'un texte engagé?

NICOLE BROSSARD En effet, j'ai déjà établi cette distinction. La poésie militante cible des causes et des responsables, elle se tient près du slogan. La poésie engagée, elle, essaie de comprendre vers où on va et pourquoi on y va, quelles sont les armes du cœur avec lesquelles on peut et veut changer la réalité. Ce que je sais intérieurement, je n'ai pas envie de le répéter dans le poème. C'est ce que dit ce poème que vous venez de lire: «je pourrais le dire», mais je ne le dirai pas parce que je le sais déjà. Dans le poème, chaque ligne est en quelque sorte un beau risque puisque le poème n'est pas là pour prouver quoi que ce soit. C'est l'effet fulgurant d'un certain agencement de mots qui donne l'impression que le poème dit vrai sans que l'on sache précisément pourquoi. Un bon poème est toujours vrai à cause de la langue.

voIX ET IMAGES Peut-être une dernière question, secondaire, concernant vos prises de position esthétiques dans les années 1970. Est-ce que cette distance tenue à l'égard d'une certaine forme de poésie militante a, d'une façon ou d'une autre, compromis le dialogue avec les poètes de l'Hexagone?

NICOLE BROSSARD Non. Cela a pu fausser le dialogue publiquement, mais sur le plan privé et amical, la discussion s'est poursuivie. Cela n'a pas affecté les liens affectifs, au sens littéraire. Il est certain que la question qui se posait pour moi et pour ma génération était celle de l'espace que nous allions occuper au présent et dans le futur. Nous avons peut-être bousculé les écrivains des générations précédentes, maladroitement, parce que nous voulions proposer autre chose. Il fallait le faire de toute façon. Nous reconnaissions tout de même la modernité là où elle était, chez nos prédécesseurs aussi, comme dans l'œuvre de Paul-Marie Lapointe, où elle était évidente, chez Roland Giguère dans l'œuvre duquel nous trouvions une forte dimension politique : «La main du bourreau finit toujours par pourrir ${ }^{10}$.» $C^{\prime}$ est étonnant comme chaque génération travaille avec peu de citations et de poèmes venus de ceux qui la précèdent. Une génération se résume à dix lignes de manifeste, trois poèmes et une obscénité! Étrange.

voix ET IMAGES De l'extérieur, en tout cas, la rupture semble complète, dès L'écho bouge beau. Cette rupture est polie, mais explicite...

NICOLE BROSSARD On était ailleurs; moi, j'étais ailleurs. Le terrain d'entente, chaleureux, c'était le politique, et non la forme, où il y avait peut-être un peu d'agacement des deux côtés. Comme on le sait, c'est parfois plus facile pour les enfants de s'amuser avec leurs grands-parents qu'avec leurs parents. Il y a un saut générationnel. Il y a toujours un lien manquant entre les générations qui donne à l'histoire le temps de se rattraper.

VoIX ET IMAGES Nous vous prendrons au mot: considérez-vous avoir des petitsenfants littéraires?

NICOLE BROSSARD En ligne directe ou non, je remarque qu'il y a une nouvelle écoute féministe et formaliste chez les jeunes femmes et, en général, un regain d'intérêt pour

$$
+++
$$

9 Nicole Brossard, «Poésie engagée», Revue de l'Université Laurentienne, vol. X, n 2, 1978, p. 121-125.

10 Roland Giguère, L'âge de la parole, Montréal, l’Hexagone, coll. «Rétrospectives», 1965, p. 17. 
la poésie des années 1970. On commence aussi à observer le fruit de l'enseignement des études féministes. Il y a aussi un nouvel imaginaire, surtout romanesque; on voit des romancières qui s'immiscent dans la peau de narrateurs masculins. Il y a actuellement au Québec une génération qui ne lit pas seulement des auteurs français, qui connaît Mandelstam, Pessoa, Pizarnik, Mario Luzi, Szymborska, Ashbery. Une génération qui s'intéresse à la traduction, ce qui pour moi est un indice vital d'une littérature en pleine maturité.

VoIX ET IMAGES Et en poésie?

NICOLE BROSSARD Il y a au Québec de nouvelles voix très intéressantes, très variées: Kim Doré, Philippe More, Isabelle Gaudet-Labine, Maggie Roussel, François Guérette, Marie-Josée Charest, François Turcot, pour ne nommer que de très jeunes poètes.

VoIX ET IMAGES Tant à l'étranger qu'au Québec, vous avez multiplié les participations aux événements qui donnaient une place publique à la poésie et, plus largement, au littéraire (colloques, festivals, marchés du livre, résidences d'écrivain, causeries). On sait notamment combien ont été importantes dans votre parcours les colloques $L a$ femme et l'écriture (1975), la Conférence des femmes-écrivains des Amériques (1978) et Les femmes et les mots (1983). Quels sont les rendez-vous pour nous inconnus qui ont pour vous été déterminants? Les événements plus petits peut-être, plus curieux? NICOLE BRosSARD Au début des années 1980, à Toronto, il y a eu une série de rencontres qui s'appelait Writers in dialogue où deux écrivaines s'entretenaient. J'ai eu la chance d'être invitée en dialogue avec Adrienne Rich. Cela a été un moment très important dans ma vie, parce que la rencontre a été très belle, mais aussi parce qu'elle a conduit à une présence plus suivie de ma réflexion et de mes écrits au Canada anglais et aux États-Unis. Puis il y a eu une rencontre, en 1985, avec le poète new-yorkais Charles Bernstein et la découverte du groupe Language Poets à l'occasion d'un colloque à Vancouver qui a aussi eu un impact semblable sur mes échanges avec l'avantgarde littéraire de New York et de San Francisco. Et puis une conférence au Colorado sur le roman des Amériques et les cinq Foires du livre féministe à Londres, Oslo, Montréal, Barcelone et Amsterdam. Bien évidemment, il y a ma rencontre avec le monde hispanophone, en particulier avec l'Argentine, le Mexique et l'Espagne.

voIX ET IMAGES Dans «S'offrir ", essai publié dans Double impression, vous déclariez que «le lyrisme est la poubelle du vingtième siècle ${ }^{11}$ ». Le lyrisme semble y incarner une tradition poétique, rhétorique, artificielle, masculine. C'est un artefact du vieil humanisme: « attention le lyrisme est formel trop tard trop art les ateliers lyriques font salle comble remplie de petits garçons de grands poètes attention le lyrisme c'est pour hier». NICOLE BROSSARD Derrière cette phrase, «le lyrisme est la poubelle du vingtième siècle», il y avait surtout un malaise par rapport à l'emploi, en poésie, du «je» qui s'étend partout. «S'offrir» est un texte qui, sous couvert d'une attaque contre le lyrisme, joue à fond la carte lyrique.

voIX ET IMAGES Diriez-vous qu'il y a tout de même eu chez vous une répudiation du lyrisme?

$$
++
$$

11 Nicole Brossard, "S'offrir», Double impression. Poèmes et textes 1967-1984, Montréal, l'Hexagone, coll. «Rétrospectives», 1984, p. 128. 
NICOLE BROSSARD Oui, au début, mais je sais trop bien à quel point une forme lyrique a l'avantage de dérouler devant nous les possibilités d'un paysage, d'un voyage intime et qu'il n'en tient qu'à nous par la suite de fragmenter, de découper autrement. Le sens apparent par exemple est un texte lyrique. Langues obscures aussi, dont le sujet est justement la place et le déploiement du «je» dans le poème.

VoIX ET IMAGES Mais à une certaine époque, dans les années 1970?

NICOLE BROSSARD Oui... Dans la façon d'énoncer, j'ai certainement remis en question un certain lyrisme lié en grande partie à l'emploi facile du «je» et j'ai toujours eu une résistance au journal intime, à l'anecdotique et à l'autobiographie. On le constate dans plusieurs livres ou articles de ces années-là. C'est un questionnement qui va aussi revenir plus tard, dans Amantes et, bien sûr, dans Langues obscures.

voıX et IMAGES En 1986, vous définissiez la poésie comme «le lieu de l'inénarrable ${ }^{12}$ ». Or, on retrouve sans doute, dans votre production récente, dont l'exemple le plus probant serait Piano blanc, des bribes de narration. La poésie peut-elle raconter?

NICOLE BROSSARD La poésie a toujours raconté, bien qu'un peu moins à partir du $\mathrm{XIX}^{\mathrm{e}}$ siècle. On racontait mais guidé par les lois du genre et de la rhétorique. Aujourd'hui, il n'y a plus de contraintes autres que celles que chacun et chacune s'impose. Qu'est-ce qui reste? Et ce qui reste est-il nécessairement prosaïque parce qu'il n'est plus déterminé par des critères rhétoriques? Je crois qu'il faut, à la lecture d'un texte moderne, être attentif à des choses très matérielles, qui traduisent une habileté poétique : à la syntaxe, à la grammaire, au symbolisme des mots. Quelle dose de mots à forte teneur symbolique faut-il pour faire basculer un texte du prosaïque au poétique? Qu'est-ce qui désorganise à ce point le phrasé prosaïque pour que son contenu se transforme en matière poétique?

voIX ET IMAGES Dans vos œuvres, et particulièrement dans les plus récentes, se déploie tout un imaginaire de la lenteur. Que représente-t-elle pour vous?

NICOLE BROSSARD Votre question est d'autant plus intéressante que chez moi un des mots clés à été celui de "vitesse». Vous voyez sans doute cette lenteur dans l'usage de l'imparfait, de bribes de prose insérées ici et là, moins d'ellipses peut-être, oui, tout cela donne sans doute une impression de ralenti.

VoIX ET IMAGES Oui. Et le terme lui-même revient beaucoup.

NICole BRosSARD D'abord, écrire c'est, par essence, ralentir la pensée ou la parole. Peu importe que l'on compte en secondes ou nanosecondes, il y a dans l'écriture un voyage dans le temps. Parfois on y oublie un mot puis on le retrouve mais, entretemps, il a voyagé et est déjà chargé d'un nouveau parcours. Quoi qu'il en soit, j'associe aussi la lenteur à des bribes de prose narrative qui atténuent la tension sémantique en la répartissant sur plusieurs mots dans la phrase.

VoIX ET IMAGES N'y a-t-il pas aussi certains objets de vision qui ralentissent la subjectivité et l'écriture?

NICOLE BROSSARD Oui : la mer, l'agonie, la mort obligent à des ralentis. C'est donc une bonne raison pour ne pas faire encore le deuil de la vitesse et du fragment. 
VoIX ET IMAGES La question du sacré semble absente de vos recueils. Il y a là quelque chose de fascinant parce qu'il est très difficile d'éviter son vocabulaire, ne serait-ce que par analogie (l'expérience comme épiphanie, par exemple).

NICOLE BROSSARD Je n'arrive pas à intégrer le mot «sacré» dans mon vocabulaire. «Âme» est cependant un mot que je n'hésite pas à utiliser pour désigner une dimension vaste de l'être. Et quand il m'arrive d'utiliser le mot «spirituel», c'est au sens d'énergie et de vibrations, un peu en pensant à ce «tout ange est terrible» de Rilke. Nous vivons dans une culture où nous sommes hypertrophiés affectivement, un peu développés intellectuellement et absolument sous-développés spirituellement. Les religions, en tout cas les trois monothéismes, n'ont rien à voir avec le développement spirituel, qui est infiniment plus raffiné. Disons que, d'une manière générale, nous manquons de silence et de recueillement.

voIX ET IMAGES Vous allez même jusqu'à écrire, dans Installations, que «le pape est un tueur/assez précis ${ }^{13} »$.

NICOLE BROSSARD Ces vers résument assez bien mon rapport au pouvoir religieux et au danger qu'il représente pour les femmes dont on exige un corps et un esprit soumis à l'autorité masculine. Les décisions du pape continuent de coûter la vie à beaucoup de femmes.

VoIX ET IMAGES Autre grande absente - toutes proportions gardées - dans votre œuvre: la nature. Dans la préface à $D^{\prime}$ aube et de civilisation, Louise Dupré rappelle comme la ville a une place importante dans votre poésie. Dans Amantes, vous écrivez par ailleurs : «je traverse/les villes sans simuler la nature car/je suis si civilisée face à la mer ${ }^{14}$ ». Et lorsqu'on tombe dans Piano blanc sur un vers comme "Quant aux arbres ", on demeure presque étonnés de croiser des arbres... Il y a bien sûr la mer, le désert, mais où sont passés les arbres, où est la nature? Qu'est-ce qui lui est arrivée NICOLE BROSSARD C'est peut-être un amour de l'abstraction chez moi qui vous donne cette impression d'absence. La ville est certainement un lieu d'effervescence que j'ai privilégié. Je me souviens très bien de l'extrait que vous citez car je pensais au lien privilégié, dit-on, que les femmes ont avec la nature et je me disais que non, je n'allais pas faire semblant de ne pas savoir ce que la civilisation imprime en nous; je pensais aussi qu'on n'est jamais intact de culture même quand on regarde la mer, les étoiles, la montagne. Et disons-le, en poésie, la nature se cache dans les grands symboles que sont les noms d'animaux, d'arbres, les couleurs, les quatre éléments. Il est vrai que j'ai tendance à faire abstraction d'un certain nombre de réalités... et pourtant j'ai l'impression que, dans mes poèmes, la nature est là dans son plus simple appareil : soleil, neige, mer, îles, eau, cosmos et neurones, sans oublier le corps. J'emploie souvent le mot "paysage » qui, lui, fait synthèse de tous les arbres que vous voulez! Et il y a bien quelques palmiers, hibiscus et roses ici et là, non?

voIX ET IMAGES En s'arrêtant aux titres de vos œuvres poétiques, on remarque que nombre d'entre eux indiquent une orientation, une situation (temporelle, spatiale) ou évoquent le littéraire. Ils dirigent la lecture des œuvres en répondant aux

$$
++
$$

13 Nicole Brossard, Installations : avec et sans pronoms, Trois-Rivières/Pantin, Écrits des Forges/Castor astral, 1989, p. 66. 14 Nicole Brossard, Amantes, Montréal, Quinze, coll. «Réelles», 1980, p. 107. Brossard souligne. 
questions: «où?», «quand?», voire «quoi?»... mais, Amantes mis à part, il n'y a pas de titre qui réponde d'avance au « qui ? ». Le point de départ, pour le lecteur, n'est donc pas une figure, une subjectivité, un énonciateur.

NICOLE BROSSARD Bien sûr, il y a toujours un sujet derrière le texte. Dans mon cas, je crois cependant que ce sujet ne s'intéresse pas à l'anecdote de son propre sujet et préfère se glisser dans l'étrangeté, l'altérité, souvent elle-même altérée par la métaphore d'une langue étrangère. De là sans doute cette phrase de La capture du sombre : "Restez vivant comme quelqu'un qui n'est pas vous».

VoIX ET IMAGES Question indiscrète et abrupte, pour conclure, qui écarte momentanément les définitions que vous avez données de ces deux termes. À choisir: le réel ou la fiction?

NICOLE BROSSARD Je crois que le réel en général n'est pas très intéressant et que seulement ce qui arrive à notre corps a une valeur sémantique. Pour le reste, tout n'est qu'imagination, traduction, interprétation pour le meilleur et pour le pire, bien sûr. Il ne faut vraiment pas se tromper de narration en amour ou devant la perte. Une partie de la joie de vivre est dans notre pouvoir d'invention, de fiction. On ne sait que trop que le corps est un boulet pour les êtres virtuels que nous sommes. Et pourtant un peu de soleil, des huîtres et du vin! 